\title{
Simultaneous, Real-Time Imaging of Intracellular Calcium and Cellular Traction Force Production
}

BioTechniques 33:358-364 (August 2002)

\author{
Andrew D. Doyle and \\ Juliet Lee \\ University of Connecticut, \\ Storrs, CT, USA
}

\section{INTRODUCTION}

Over the past several years, there has been an explosion of interest in understanding how cells sense and respond to their physical environment $(5,15)$. This is partly due to the increasing number of studies that show cellular behavior is affected by various mechanical stimuli. Cells can respond to speed or direction of fluid flows (22), mechanical stretching (28), and even cytoskeletal geometry (4). In addition, the behavior of moving cells is altered by the rigidity of the substratum (21) and changes in cytoskeletal tension (17). Furthermore, the emergence of new fields such as tissue engineering and gravitational biology has also underscored the importance of investigating how cells sense and respond to changes in their physical environment. To date, there have been relatively few studies of mechano-chemical signaling mechanisms compared with those of biochemical signaling. However, as interest in mechano-chemical signaling grows, so will the demand for techniques to study this process. Here we report the development of a new method for studying mechano-chemical transduction and demonstrate its use in the study of cell movement.

Moving cells generate and utilize mechanical forces for movement $(16,29)$. For example, polymerization of actin is involved in generating protrusive forces at the cell front, while contractile forces are limited to the rear to assist with retraction and forward movement of the cell body. However, it is not understood how cytoskeletal force production is spatially and tem- porally regulated during movement. Recently, it has been shown that coordination of protrusion with retraction is mediated by stretch-activated calcium channels (SACs) in rapidly moving fish epithelial keratocytes (17). SACs are known to function as mechano-chemical signal transducers in a variety of systems by triggering a transient increase in intracellular calcium $\left(\left[\mathrm{Ca}^{2+}\right]_{\mathrm{i}}\right)$ in response to an applied force $(10,24)$. In keratocytes, SACs are activated in response to an increase in cytoskeletal tension that occurs when retraction of the rear cell margin is impeded. The resulting calcium transient triggers retraction of the rear, allowing cell movement to continue. Thus, SACs provide a positive feedback mechanism that is important for maintaining a rapid mode of movement in keratocytes. The movement of other rapidly moving cell types may also involve the activation of SACs, since their movement has been shown to depend on transient increases in calcium that result from an initial influx of extracellular calcium $(23,25)$.

To investigate the mechano-chemical regulation of cell movement, it is necessary to monitor changes in cytoskeletal contractility and intracellular calcium simultaneously. A number of "traction force" assays have been developed to study how cells utilize contractile forces for movement. These generally consist of a flexible surface or substratum on which cells can adhere and move. Contractile forces generated within the cytoskeleton are transmitted to the substratum via cell adhesions and become manifest as wrinkles or deformations in the plane of the substratum. A range of materials 
has been used for traction force assays, such as films of cross-linked silicone oil $(3,11,19)$, polyacrylamide gels $(30)$, silicone elastomer (1), and even micromachined surfaces embedded with quartz cantilevers (9). However, none of these assays has yet been combined with calcium imaging, possibly because of problems of substratum thickness, autofluorescence, and optical clarity of the substratum. These factors are particularly limiting for calcium imaging in single cells because this requires the use of high-magnification, oil-immersion objectives that have short working distances, in conjunction with low-light-level fluorescence imaging. Another disadvantage of many traction force assays is their inability to register relatively small traction forces generated by rapidly moving cells (19). This will also limit the temporal resolution of the assay, since stiffer substrata may not register small changes in traction force generation that occur over a time period of several seconds.

We have developed a new traction force assay using low concentrations of gelatin that can detect changes in traction force generation that accompany calcium transients in moving fish keratocytes. The gelatin traction force assay consists of a thin, highly elastic, gelatin substratum that is embedded with small, fluorescent marker beads. One of the major advantages of this assay is that gelatin substrata are transparent and can be made thin enough to perform calcium imaging using high-magnification objectives. In addition, the temporal resolution of gelatin substrata is significantly greater than other traction force assays. This is especially im portant for detecting changes in rate of traction force production that accompany rapid, transient increases in $\left[\mathrm{Ca}^{2+}\right]_{i}$. Some further advantages of gelatin substrata include their ease of preparation and the ability to vary their elasticity reproducibly over a range of values so that these substrata may be used to detect traction forces generated by a variety of cell types.

Here we describe the preparation of the gelatin traction force assay, its elastic properties, and its use in combination with calcium imaging in moving fish keratocytes. We also demonstrate how this assay can be used to study the mechano-chemical regulation of cell movement.

\section{MATERIALS AND METHODS}

\section{Preparation of Cells for Calcium Imaging}

Fish epithelial keratocytes were cultured from Molly fish Poecillia sphenops, as described previously (18). L-15 culture medium was used supplemented with $10 \%$ FBS, $200 \mathrm{U} / \mathrm{mL}$ penicillin-streptomycin, and $1 \mu \mathrm{g} / \mathrm{mL}$ Fungizone (all from Invitrogen, Carlsbad, CA, USA). Keratocytes were loaded with the calcium indicator Calcium Green ${ }^{\mathrm{TM}}-1$ dextran (MW $3 \mathrm{kDa}$; Molecular Probes, Eugene, OR, USA) for non-ratiometric imaging or together with the calcium insensitive dye Texas Red $^{\circledR}$ dextran (MW, 3 kDa; Molecular Probes) for ratiometric imaging, using the Influx ${ }^{\mathrm{TM}}$ pinocytic cell-loading reagent (Molecular Probes). The manufacturer's protocol was modified for use with keratocytes, as follows. Cultures were incubated in calcium and magnesium free Fish Ringer's solution (112 mM NaCl, 2 mM D-glucose, 2 $\mathrm{mM} \mathrm{KCl}, 2.4 \mathrm{mM} \mathrm{NaHCO}$ ) for $3 \mathrm{~min}$. This was replaced with $100 \mu \mathrm{L}$ (for a 35-mm petri dish) of the hypertonic loading reagent, containing $2 \mathrm{mg} / \mathrm{mL}$ calcium indicator, and incubated for 5 min in the dark. After aspiration of the loading reagent, three increasingly hypotonic solutions were added and removed from the cell culture in the following sequence. Solution no. 1 (86.7\% L-15 culture medium, $13.3 \%$ distilled water) for $30 \mathrm{~s}$, solution no. 2 (73.3\% L-15, 26.7\% distilled water) for $30 \mathrm{~s}$, and solution no. 3 (40\% L-15, $60 \%$ distilled water) for $1.5 \mathrm{~min}$. Following the removal of the final hypotonic solution, cells were placed in culture medium and allowed to recover for $15 \mathrm{~min}$ before use.

To replate cells loaded with fluorescent indicators onto gelatin substrata, they were washed twice with $\mathrm{Ca}^{2+}$ - and $\mathrm{Mg}^{2+}$-free PBS and once with trypsin (0.05\% trypsin containing $0.53 \mathrm{mM}$ EDTA; Invitrogen) before adding a 1:1 mixture of PBS and trypsin-ETDA solution. Cells were observed until epithelial sheets disaggregated complete- ly. Next, $1 \mathrm{~mL}$ serum-rich (30\% FBS) culture medium was added to cells, and they were triturated 5-10 times followed by vortex mixing for $15 \mathrm{~s}$. Serum-rich culture medium was also added to the gelatin coated cell cham bers for $30 \mathrm{~min}$ before replating. Cells were allowed to adhere to the gelatin substratum for 45-60 min at room tem perature before experimentation.

\section{Preparation of the Gelatin Traction Force Assay}

Stock gels were made with different percentages of powdered gelatin (Nabisco, Parsippany, NJ, USA) dissolved in prewarmed (approximately $\left.40^{\circ} \mathrm{C}\right) \mathrm{Ca}^{2+}$ and $\mathrm{Mg}^{2+}$-free Fish Ringer's solution. These were cooled and stored at $4^{\circ} \mathrm{C}$ until required. Nabisco gelatin has a bloom number of approximately 235 , which is an indicator of gel strength. Gels with higher bloom numbers are stronger and more rigid. Another important aspect of gelatin powder to consider is its method of preparation, because lyme-cured gelatins tend to be more rigid compared to acid-cured gelatins of a similar bloom number (8). Nabisco gelatin consists of a mixture of both acid- and lyme-cured gelatins. We generally used $2.5 \%$ and $5 \%$ gelatin solutions to make more or less deformable substrata, respectively.

Rappaport chambers were assembled by attaching a glass cylinder (diameter $=22 \mathrm{~mm}$, height $=8 \mathrm{~mm}$ ) to a $22-\mathrm{mm}^{2}$ glass coverslip (zero thickness) using Sylgard ${ }^{\circledR} 184$ (Dow Corning, Midland, MI, USA) silicone elastomer (Figure 1). Stock gels were liquefied by warming to $40^{\circ} \mathrm{C}$ in a water bath for 5-10 min. A $400-\mu \mathrm{L}$ aliquot of liquid gelatin was transferred into a Rappaport chamber and allowed to solidify at $4{ }^{\circ} \mathrm{C}$ in a humid environment for $2-24 \mathrm{~h}$. The humid environment is necessary to prevent gels from drying out, as this will effectively increase the gelatin concentration and the rigidity of the substratum. Next, $400 \mu \mathrm{L}$ of a solution containing fluorescent orange microspheres $(0.2 \mu \mathrm{m}$, Molecular Probes), diluted in distilled water and cooled to $4^{\circ} \mathrm{C}$, was added to the gelatin substratum. This solution was aspirated off im mediately, and the remaining bead 
solution was allowed to dry on to the gelatin substratum, for $1 \mathrm{~h}$ at $4^{\circ} \mathrm{C}$, under air flow. Gelatin substrata were briefly (5-15 s) warmed on a hot plate to liquefy the lower layer of gelatin. After removal from the hot plate, approximately $330 \mu \mathrm{L}$ gelatin solution were carefully aspirated from the bottom of the chamber using a small pipet tip, being careful not to disturb the surface. Gels were then rapidly cooled for $30 \mathrm{~s}$ by placing Rappaport chambers on a level metal sheet, pre-cooled to $-20^{\circ} \mathrm{C}$. This produced a thin layer of gelatin whose top surface was embedded with a monolayer of fluorescent beads. Gelatin substrata were stored at $4{ }^{\circ} \mathrm{C}$ for $1 \mathrm{~h}$ before adding $0.5 \mathrm{~mL}$ culture medium and could be used for up to $72 \mathrm{~h}$ afterward. The average thickness of gelatin substrata manufactured in this way was $40.5 \mu \mathrm{m}$ ( $\mathrm{SEM}=0.88, n=15)$. This was determined by focusing on the top surface of the coverglass, immediately below the gelatin, and on the top surface of the gelatin using a $100 \times$ objective and noting the difference in position of the fine focus control knob. Each of the smallest markings corresponds to a distance of $1 \mu \mathrm{m}$ in the $\mathrm{Z}$ direction on a Nikon TE 300 Eclipse microscope (Nikon, Melville, NY, USA).

\section{Calibration of Gelatin Substrata}

Gelatin substrata were brought to room temperature $\left(21^{\circ} \mathrm{C}-23^{\circ} \mathrm{C}\right)$ for $1 \mathrm{~h}$ before calibration. Calibrations were performed by placing a steel ball (diameter $=0.3-0.5 \mathrm{~mm}$, density $=14.95$ $\mathrm{g} / \mathrm{cm}^{3}$; Hoover Precision, East Gramby, CT, USA) on the substratum and measuring the resulting surface indentation (21). The Young's Modulus ( $\mathrm{Y}$ ) is given by $\mathrm{Y}=3\left(1-v^{2}\right) f / 4 d^{3 / 2} \mathrm{r}^{1 / 2}$, where $\mathrm{r}$ is the bead radius, $f$ is the force applied by the steel ball, $d$ is the size of the indentation, and $v$ is the Poisson ratio [assumed here to be 0.3 (20)]. Measurements were made in five random locations, and the average value for $d$ was used to calculate the Young's modulus of each substratum (Figure 2A). For temperature-controlled calibration experiments, a KT stage controller (20/20 Technologies, Whitehouse, NJ, USA) was used to regulate chamber temperature.

\section{Calcium Imaging}

Except where noted, calcium imaging was performed as described previously (14). Before imaging, culture medium was replaced with L-15 medium without serum, antibiotics, or antifungal agents to reduce background fluorescence. Calcium imaging was performed on an inverted microscope (Eclipse TE 300; Nikon) using a Plan Fluor $100 \times$ objective N.A. 1.4 (Nikon). Fluorescence excitation of both the calcium indicator and fluorescence marker beads was achieved using an FITC filter set (Chroma Technology, Brattleboro, VT, USA). A DAPI/FITC/Texas Red triple band pass filer set (Chroma Technology) was used to excite Texas Red dextran. Images were acquired using a back-illuminated, back thinned, frame transfer CCD camera (Quantix ${ }^{\circledR}$ 57; Roper Scientific, Tucson, AZ, USA) controlled by Isee Analytical Imaging Software (Isee Imaging Systems, Raleigh, NC, USA) on a Unix computer platform (SGI O; Silicon Graphics, Mountain View, CA, USA). Images of keratocytes moving on the gelatin traction force assay were collected every second, for a period of 2-3 min, and stored on the computer hard drive.

\section{Image Processing and Analysis}

Image processing was performed using Isee Analytical Imaging software. To detect changes in $\left[\mathrm{Ca}^{2+}\right]_{\mathrm{i}}$, measurements of the average fluorescence intensity were made over the cell body region (Figure 3A) in sequential images. Next, a series of difference im ages was made by dividing each pixel value in subsequent images by each pixel value in the original image. This procedure is particularly useful for identifying the subpopulation of beads that undergo displacement during the period of observation from those that do not (Figure 3, B and C). In addition, beads that undergo relatively large displacements can be easily identified and preselected for further analysis. A bead tracking algorithm was then used to obtain the coordinates of marker bead positions. Since bead displacements are proportional to the applied force, an estimate of traction force can be obtained from the Young's modulus multiplied by the size of the bead displacement. The relative increase in force production is given by the change in rate of bead displacement, following a calcium transient (Figure 3D).

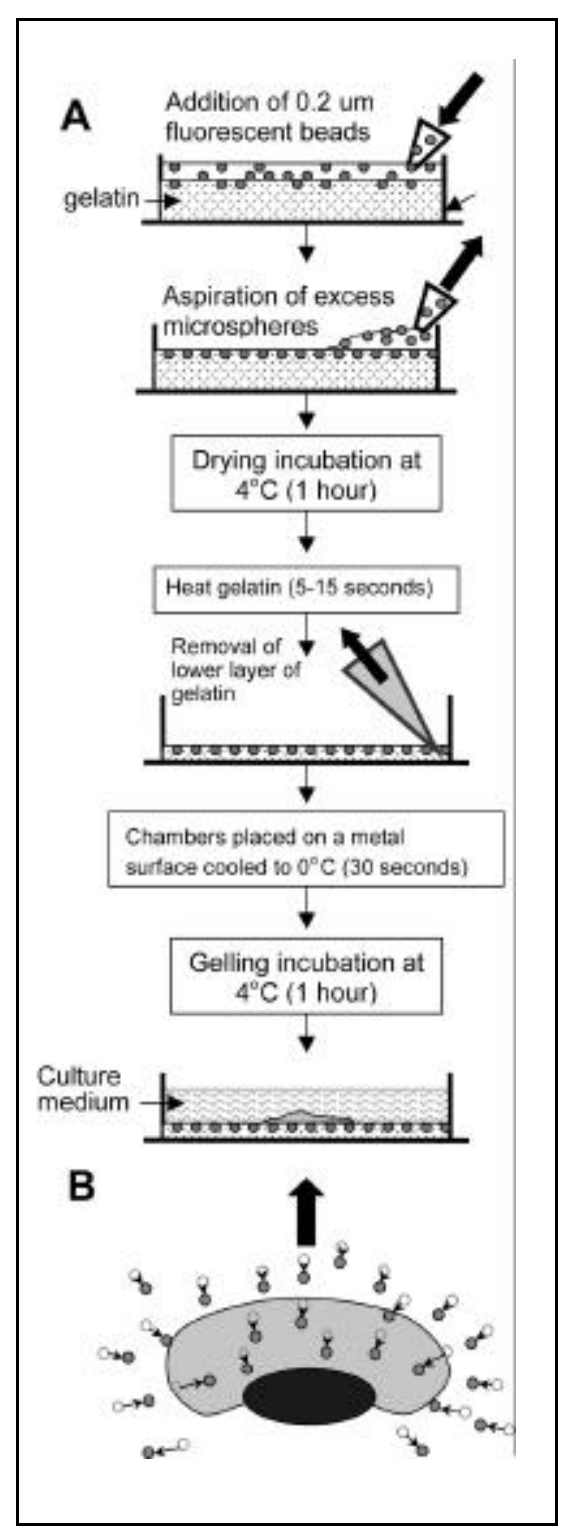

Figure 1. Preparation of gelatin traction force assay. (A) Sequence of steps showing the preparation of a thin layer of gelatin with a monolayer of small $(0.2 \mu \mathrm{m})$ fluorescent marker beads em bedded at the surface. (B) Cartoon of a fish epithelial keratocyte locomoting across a beaded gelatin substratum. Original, undisplaced bead positions (open circles) and current displaced bead positions (closed circles) are indicated. Small arrows show the direction and relative size of bead displacements, in the plane of the substratum, resulting from the application of cellular traction forces. Large, bold arrow represents direction of cell movement. 


\section{RESULTS AND DISCUSSION}

\section{Mechanical Properties of Collagen Gels}

The determination of the elastic properties of a substratum is essential, especially if a quantitative study of traction force generation is desired, since a linear response to applied force will only occur in materials whose behavior is predominantly elastic. How ever, polymeric substrata can exhibit varying degrees of non-elastic behav- ior, which if significant will preclude quantitative analysis of traction force production (19). At gelatin concentrations of $2.5 \%$ and above, and at temperatures below $26^{\circ} \mathrm{C}$, substrata are predominantly elastic. This is supported by the observation that displaced beads returned immediately to their original undisplaced positions (data not shown) following release by the cell. In experiments where microneedles were used to displace marker beads double the distance normally made by the cell (approximately $12 \mu \mathrm{m}$ ) and for 10 times
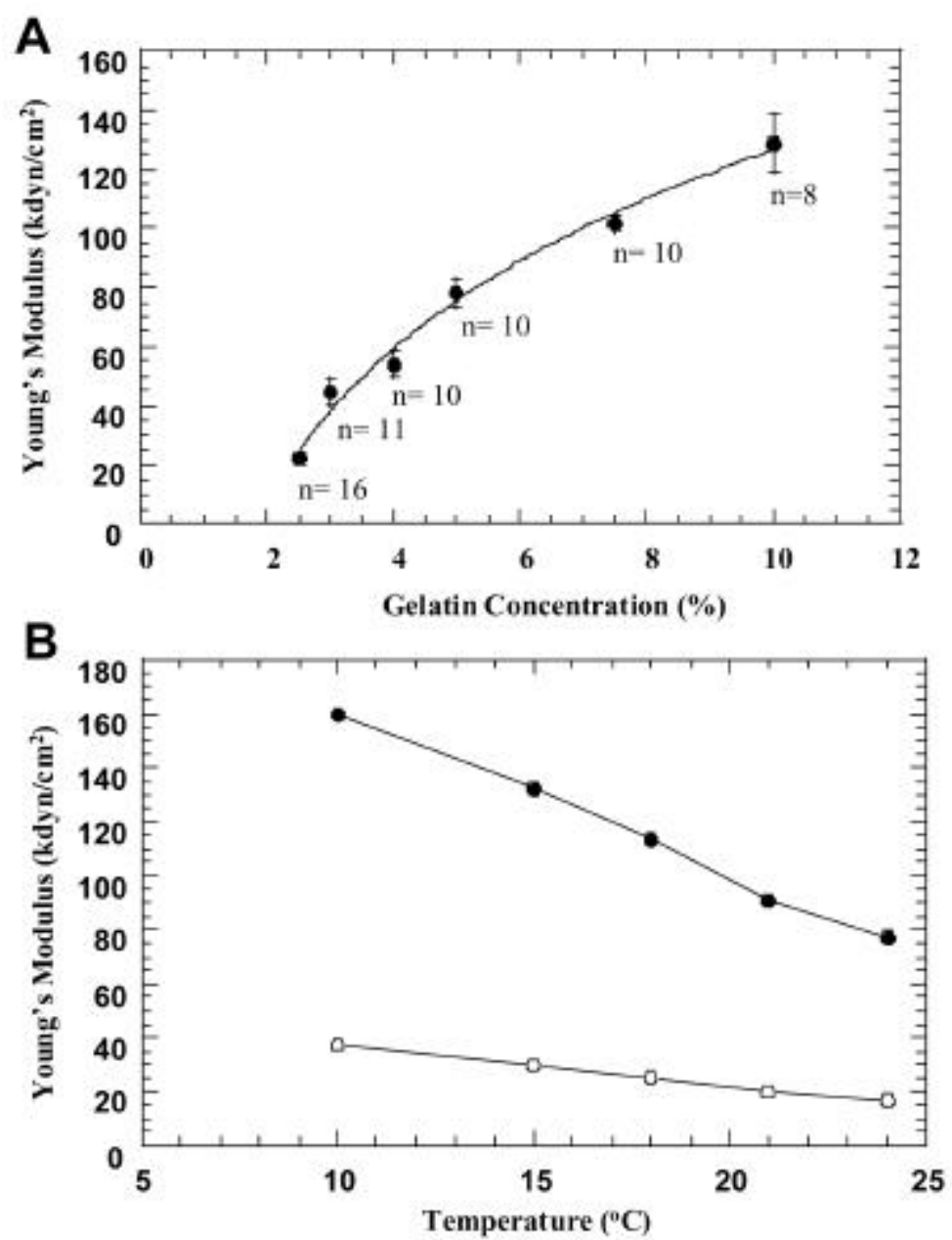

Figure 2. Effects of gelatin concentration and temperature on the elastic properties of gelatin substrata. (A) A plot of Young's modulus against gelatin concentration, measured at room temperature $\left(21^{\circ} \mathrm{C}-23^{\circ} \mathrm{C}\right)$. The mean of five measurements, made at random locations, was taken as the Young's modulus for each chamber. Data points represent the mean Young's modulus of several individual cham bers $(n)$. Error bars represent S EM (B) A plot of Young's modulus against temperature for $2.5 \%$ (open circles, $n=3$ ) and 5\% gelatin substrata (closed circles, $n=3$ ). The same percent decrease in Young's modulus occurs, with increasing temperature, for both $2.5 \%$ and $5 \%$ gelatin substrata. The SEM for data points in panel B was small and is represented by the size of the symbol. 


\section{Research Report}

the duration ( $5 \mathrm{~min}$ ), beads returned immediately to within approximately 96\% $(11.52 \mu \mathrm{m})$ of their original positions, following release.

The elasticity gelatin substrata can be varied between 22 and $160 \mathrm{kdyn} / \mathrm{cm}^{2}$ with a high degree of reproducibility by altering gelatin concentration and or temperature (Figure 2). Variations in elasticity of $2.5 \%$ gelatin substrata are negligible, when measured within a single chamber $\left(\mathrm{Y}=21.38 \mathrm{kdyn} / \mathrm{cm}^{2}, \mathrm{SEM}\right.$ $=0.45, n=20$ ) or between chambers made on the same day $(\mathrm{Y}=24.16$ $\mathrm{kdyn} / \mathrm{cm}^{2}, \mathrm{SEM}=0.75, n=6$ ). A small but insignificant increase in variation of elasticity is seen between chambers made on separate days $(\mathrm{Y}=22.35$ $\mathrm{kdyn} / \mathrm{cm}^{2}, \mathrm{sEM}=2, n=16$ ). Another im portant feature of gelatin substrata is the constancy of their elastic properties under experimental conditions over an extended period of time. For example, no detectable change in the Young's modulus was observed $(\mathrm{Y}=25.83 \mathrm{kdyn} / \mathrm{cm}$, $\mathrm{SEM}=0.24, n=40$ ) within $2.5 \%$ gelatin substrata that were submerged in culture
Table 1. Elasticity of Different Types of Traction Force Assays

\begin{tabular}{|lcc|}
\hline Substratum & $\begin{array}{c}\text { Young's Modulus } \\
\text { (kdyn/cm } \mathbf{~})\end{array}$ & Reference \\
\hline Gelatin & $22-160$ & \\
Polymerized Silicone & $5(\mathrm{C})$ & 19 \\
$\quad$ Viscoelastic & $20(\mathrm{C})$ & 26 \\
Elastic & 6000 & 27 \\
Polyacrylamide Gel & $140-300$ & 21 \\
Polyacrylamide Gel & $120-10000$ & 1 \\
Sylgard 184 & & \\
C = Compliance (dyn/cm) & & \\
\hline
\end{tabular}

medium and maintained at room tem perature over a $24-\mathrm{h}$ period. Although the type of gelatin used in this study liquefies above $30^{\circ} \mathrm{C}$, this should not pose a significant problem for working with cells at $37^{\circ} \mathrm{C}$, since other types of gelatin with increased thermal stability can be used for this type of assay (2).

Gelatin substrata are generally more deformable than existing traction force

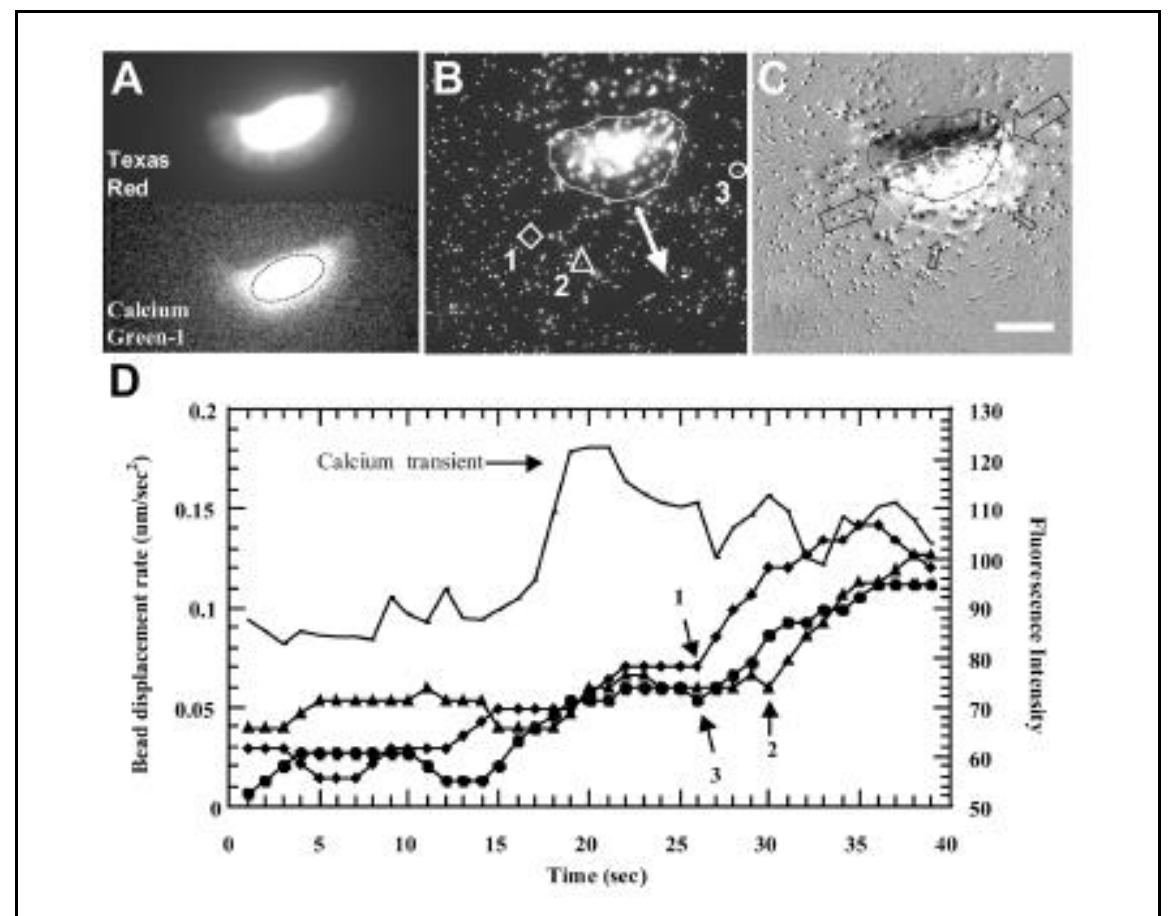

Figure 3. Combined calcium and traction force imaging using gelatin substrata. (A) Fluorescence images of a moving keratocyte co-loaded with Texas Red dextran (top panel) and the calcium indicator Calcium-Green 1-dextran (bottom panel) on a $2.5 \%$ gelatin substratum without beads. Fluorescence intensity of the calcium indicator was measured within the oval region indicated (black line). (B) Fluorescence image of a moving (arrow) keratocyte (white outline) loaded with both Texas Red dextran (not shown) and calcium indicator (shown) on a beaded gelatin substratum. Numbers 1-3 with symbols indicate beads that were tracked over the period shown in panel D. (C) A difference image generated from images of the keratocyte in panel B taken at the beginning (black outline) and end (white outline) of the observation period in panel D. Bead displacements are shown by the distance between black (original bead position) and corresponding white (final bead position) regions. The relative size and direction of bead displacements are indicated by the open black arrows. (D) A double plot of calcium indicator fluorescence and change in rate of bead displacement over time. A marked increase in rate of bead displacement occurs about $10 \mathrm{~s}$, following a calcium transient (indicated) for all three beads (1-3, arrows) shown in panel B. Changes in bead displacement rates were obtained by averaging bead displacement rates occurring within successive, overlapping 10 -s intervals. $\mathrm{Bar}=10 \mu \mathrm{m}$. 
assays (Table 1), so they are ideally suited for the study of rapidly moving cell types that typically produce smaller traction forces (19). In addition, the greater sensitivity of gelatin substrata allows detection of changes in traction force production over a period of several seconds. Thus, the temporal resolution of gelatin substrata is sufficient to register changes in traction force production that occur during a calcium transient. Furthermore, the elastic modulus of "stiffer" gelatin substrata is sim ilar to other traction force assays (Table 1). Thus, gelatin traction force assays can be adjusted to detect relatively large traction forces generated by slow moving cell types. The major advantage of this is that it will allow a more direct comparison to be made between the magnitudes of forces generated by both fast- and slow-moving cell types. Such a comparison is not generally possible with most traction force assays because their useful range of elasticity restricts their use to either fast- or slowmoving cells.

\section{Evaluation of Gelatin Substrata for Use with Calcium Imaging}

The most important features of a substratum that will be used as a traction force assay in combination with calcium imaging are its thickness, transparency, and flexibility. For highresolution calcium imaging, the thickness of the substratum must be less than the working distance of the objective. Gelatin substrata are generally $40 \mu \mathrm{m}$ in thickness, which is well within the working distance $(130 \mu \mathrm{m})$ of the $100 \times$ objective used here. However, the transparency and autofluorescence of the substratum are especially important factors for determining whether a traction force assay will be suitable for combined use with calcium imaging. First, substratum transparency will influence transmission of light at excitation and emission wavelengths, while the generation of background fluorescence will decrease image contrast. Second, calcium imaging in single motile cells requires the reduction of incident fluorescence light to $1 \%-10 \%$ to minimize any phototoxic effects during continuous, prolonged periods (2-3 $\min$ ) of observation. Therefore, increas- ing the throughput of fluorescence light cannot be used to compensate for poor substratum transparency or autofluorescence. To assess different substrata in terms of their background fluorescence, we measured the average fluorescence intensity within regions of the background (B) and of the cell (F) during calcium imaging experiments. For each type of substratum, we obtained a value of image contrast $(\mathrm{C})$ according to the formula, $\mathrm{C}=(\mathrm{F}-\mathrm{B}) /(\mathrm{F}+\mathrm{B})$. Compared with cells imaged on a glass surface $($ mean $=0.28$, SEM $=0.05, n=18), \mathrm{im}$ age contrast was reduced by $26 \%$ on gelatin $($ mean $=0.19$, SEM $=0.03, n=$ 25 ) and by $50 \%$ on Sylgard (mean = $0.14, \operatorname{SEM}=0.02, n=19$ ). Although there is a reduction in fluorescence signal with gelatin substrata, this is significantly less than observed with Sylgard substrata that have recently been used for dual fluorescence and traction force imaging at sites of focal adhesion (1). Therefore, gelatin substrata could also be suitable for use in conjunction with other types of fluorescence microscopy besides calcium imaging.

To detect changes in traction force production that accompany transient increases in $\left[\mathrm{Ca}^{2+}\right]_{i}$, bead displacements must occur on the same time scale as the calcium signaling events being studied. For real-time imaging, it is also desirable that bead displacements in the substratum are sufficiently large that they can be observed as calcium transients occur. This is particularly useful in determining if an experimental perturbation, such as the photorelease of a caged compound (14), is producing an effect during the experiment. The highly elastic nature of gelatin substrata allows observable bead displacements (1 $\mu \mathrm{m})$ to be detected within approximately $5 \mathrm{~s}$ (Figure 3D), which is less than the duration of a typical calcium transient (10-15 s). In contrast, it can take 5-10 min for the same displacement to be observed using other, more rigid elastic traction force assays (Table 1). Bead tracking algorithms can detect nanometer-scale bead displacements and so could be used to measure changes in traction force generation during a calcium transient that otherwise would not be observable. This will be particularly useful when performing simultaneous calcium and traction force imaging on 
stiffer gelatin substrata. Although it has previously not been possible to track the movements of many beads simultaneously, in real time, the increased speed and computational power of today's computers should allow accurate detection of very small bead displacements during an experiment.

\section{Combined Calcium and Traction Force Imaging as Applied to the Study of Mechano-Chemical Signal- ing in Moving Cells}

In fish keratocytes, SACs have been shown to serve as mechano-chemical regulators of cell movement, by allow ing cells to sense and respond to increased cytoskeletal tension that occurs when retraction is impeded (17). Although stretch-induced calcium transients have been shown to trigger retraction, it is not known which downstream mechanisms are involved. A longstanding possibility is that a calcium induced increase in cytoskeletal contractility could cause retraction (6). However, it is also possible that calcium-dependent adhesion disassembly mechanisms could play a role in retraction $(12,13)$. Using the gelatin traction force assay in conjunction with calcium imaging, we have demonstrated directly that an increase in traction force generation occurs approximately $4 \mathrm{~s}$ following a calcium transient (Figure 3D). In addition, the change in rate of bead displacement suggests that the rate of traction force production is increased 2- to 6fold. Thus, combined calcium and traction force imaging will be useful in distinguishing between different mechanisms of calcium induced retraction, since it should be possible to selectively inhibit either one of these processes and then to observe how retraction is affected following a calcium transient.

The gelatin traction force assay will permit a quantitative analysis to be made of the changes in traction force production that accompany changes in $\left[\mathrm{Ca}^{2+}\right]_{\mathrm{i}}$, since it undergoes elastic deformations in the plane of the substratum without wrinkling (7). Furthermore, this assay could provide a general means of studying mechanochemical signaling, since it can be used in combination with other fluorescence techniques and with various cell types.

\section{ACKNOWLEDGMENTS}

This work was supported by National Science Foundation grant no. MCB0114231 to J.L.

\section{REFERENCES}

1.Balaban, N.Q., U.S. Schwartz, D. Riveline, P. Goichberg, G. Tzur, I. Sabany, D. Mahalu, S. Safran, et al. 2001. Force and focal adhesion assembly: a close relationship studied using elastic micropatterned substrates. Nat. Cell Biol. 3:466-472.

2.Bigi, A., G. Cojazzi, S. Panzavolta, K. Rubini, and N. Roveri. 2001. Mechanical and thermal properties of gelatin films at different degrees of glutaraldehyde crosslinking. Biomaterials 22:763-768.

3.Burton, K. and D.L. Taylor. 1997. Traction forces of cytokinesis measured with optically modified elastic substrata. Nature 385:450454.

4.Chen, C.S., M. Mrksich, S. Huang, G.M. Whitesides, and D.E. Ingber. 1997. Geometric control of cell life and death. Science 276:1425-1428.

5.Chicurel, M.E., C.S. Chen, and D.E. Ingber. 1998. Cellular control lies in the balance of forces. Curr. Opin. Cell Biol. 10:232-239.

6.Citi, S. and J. Kendrick-Jones. 1987. Regulation of non-muscle myosin structure and function. Bioessays 7:155-159.

7.Dembo, M. and Y.-L. Wang. 1999. Stresses at the cell-to-substrate interface during locomotion of fibroblasts. Biophys. J. 76:2307-2316.

8.Djabourov, M., J.-P. Lechaire, and F. Gaill. 1993. Structure and rheology of gelatin and collagen gels. Biorheology 30:191-205.

9.Galbraith, C.G. and M.P. Sheetz. 1997. A micromachined device provides a new bend on fibroblast traction forces. Proc. Natl. Acad. Sci. USA 94:9114-9118.

10.Glogauer, M., J. Ferrier, and C.A.G. McCullouch. 1995. Magnetic fields applied to collagen-coated ferric oxide beads induce stretch-activated $\mathrm{Ca}^{2+}$ flux in fibroblasts. Am. J. Physiol. 269:C1093-C1104

11.Harris, A.K., P. Wild, and D. Stopak. 1980. Silicone rubber substrata: a new wrinkle in the study of cell locomotion. Science 208:177179.

12.Hendey, B., C.B. Klee, and F.R. Maxfield. 1992. Inhibition of neutrophil chemokinesis on vitronectin by inhibitors of calcineurin. Science 258:296-299.

13.Huttenlocher, A., S.P. Palecek, Q. Lu, W. Zhang, R.L. Mellgren, D.A. Lauffenberger, M.H. Ginsberg, and A.F. Horwitz. 1997. Regulation of cell migration by the calciumdependent protease calpain. J. Biol. Chem. 272:32719-32722

14.Ishihara, A., K. Gee, S. Schwartz, K. Jacobson, and J. Lee. 1997. Photoactivation of caged compounds in single, living cells: an application to the study of cell locomotion. BioTechniques 23:268-274.

15.Janmey, P.A. 1998. The cytoskeleton and cell signaling: component localization and me- chanical coupling. Physiol. Rev. 78:763-781.

16.Lauffenberger, D.A. and A.F. Horwitz 1996. Cell migration: a physically integrated molecular process. Cell 84:359-369.

17.Lee, J., A. Ishihara, G. Oxford, B. Johnson, and K. Jacobson. 1999. Regulation of cell movement is mediated by stretch-activated calcium channels. Nature 400:382-386.

18.Lee, J., A. Ishihara, A. Theriot, and K. Jacobson. 1993. Principles of locomotion for simple-shaped cells. Nature 362:167-171

19.Lee, J., M. Leonard, T. Oliver, A. Ishihara, and K. Jacobson. 1994. Traction forces generated by locomoting keratocytes. J. Cell Biol. 127:1957-1964.

20.Li, Y., Z. Hu, and C. Li. 1993. New method for measuring Poisson's ratio in polymer gels. J. Appl. Polymer Sci. 50:1107-1111.

21.Lo, C.-M., H.-B. Wang, M. Dembo, and Y.L. Wang. 2000. Cell movement is guided by the rigidity of the substrate. Biophys. J. 79:144-152

22.Malek, A.M. and S. Izumo. 1996. Mechanism of endothelial cell shape change and cytoskeletal remodeling in repsonse to fluid shear stress. J. Cell Sci. 109:713-726.

23.Marks, P.W. and F.R. Maxfield. 1990. Transient increases in cytosolic free calcium appear to be required for the migration of adherent human neutrophils. J. Cell Biol. 110:43-52.

24.Naruse, K. and M. Sokabe. 1993. Involvement of stretch-activated ion channels in $\mathrm{Ca}^{2+}$ mobilization to mechanical stretch in endothelial cells. Am. J. Physiol. 264:1037-1044.

25.Nebl, T. and P.R. Fisher. 1997. Intracellular $\mathrm{Ca}^{2+}$ signals in Dictyostelium chemotaxis are mediated exclusively by $\mathrm{Ca}^{2}+$ influx. J. Cell Sci. 110:2845-2853.

26.Oliver, T., M. Dembo, and K. Jacobson. 1995. Traction forces and locomoting cells. Cell Motil. Cytoskeleton 31:225-240.

27.Pelham, R.J. and Y.-L. Wang. 1999. High resolution detection of mechanical forces exerted by fibroblasts on the substrate. Mol. Biol. Cell 10:935-945.

28.Pender, N. and C.A.G. McCullouch. 1991 Quantitation of actin polymerization in two human fibroblast sub-types responding to mechanical stretching. J. Cell Sci. 100:187-193.

29.Schwarzbauer, J.E. 1997. Cell migration: may the force be with you. Curr. Biol. 7:R292R294.

30.Wang, Y.-L. and R.J. Pelham. 1998. Preparation of a flexible porous polyacrylamide substrate for mechanical studies of cultured cells. Methods Enzymol. 298:489-496.

Received 28 November 2001; accepted 25 April 2002.

\section{Address correspondence to:}

Dr. Juliet Lee

Department of Molecular and Cell Biology

75 North Eagleville Rd.

$U-3125$

University of Connecticut

Storrs, CT 06269, USA

e-mail: jlee@uconnvm.uconn.edu 